\title{
Pharmacogenomics of CYP3A5 Polymorphism: Predicting Dose-adjusted Trough Levels of Tacrolimus in South Indian Renal Transplant Patients
}

\author{
Sarasamma $\mathbf{S}^{1^{*}}$, Gracious $\mathbf{N}^{2}$, Nair $\mathbf{S S}^{2}$ and Radhakrishnan $\mathbf{R}^{3}$ \\ ${ }^{1}$ Srinivasa Ramanujam Institute for Basic Sciences, RGCB-Biolnnovation Centre, Thiruvananthapuram 695585, Kerala, India \\ ${ }^{2}$ Department of Nephrology, Medical College Hospital, Thiruvananthapuram, Kerala, India \\ ${ }^{3}$ Laboratory Medicine \& Molecular Diagnostics, Rajiv Gandhi Centre for Biotechnology, Poojappura, Thiruvananthapuram 695014, Kerala, India
}

*Corresponding author: Sarasamma S, Srinivasa Ramanujam Institute for Basic Sciences, RGCB-Bio-Innovation Centre, Thiruvananthapuram 695585, Kerala, India, Tel: 919496775942; Email: sreejakarthik@hotmail.com

Received date: Sep 05, 2016; Accepted date: Sep 15, 2016; Published date: Sep 22, 2016

Copyright: (c) 2016 Sarasamma S, et al. This is an open-access article distributed under the terms of the Creative Commons Attribution License, which permits unrestricted use, distribution, and reproduction in any medium, provided the original author and source are credited.

\begin{abstract}
Tacrolimus is a potent immunosuppressant clinically used for the long term treatment of antirejection of transplanted organs in liver and kidney transplant recipients although dose optimization is often poorly managed. So far, no study has been carried out in the South Indian kidney transplant patients. The objective of this study was to evaluate the potential influence of a functional polymorphism in CYP3A5*3 gene on tacrolimus physiological availability/dose ratio in South Indian renal transplant patients. Twenty five renal transplant recipients receiving tacrolimus were enrolled in this study. Their body weight, drug dosage, and therapeutic concentration of tacrolimus were observed. All patients were on a standard immunosuppressive regime of tacrolimus-mycophenolate mofetil (Immunosuppressant) along with steroids at a starting dose of $0.1 \mathrm{mg} / \mathrm{kg} / \mathrm{day}$ tacrolimus. CYP3A5 genotyping was performed by PCR followed with RFLP. Confirmation of RFLP analysis and variation in the nucleotide sequence of CYP3A5 3 gene were determined by direct sequencing using a validated automated genetic analyzer. A significant association was found between tacrolimus dose $/ \mathrm{kg} / \mathrm{d}$ and CYP3A5 gene (A6986G) polymorphism in the study population. The CYP3A5 $* 1 /{ }^{*} 1,{ }^{*} 1{ }^{*} 3$ and ${ }^{*} 3 /{ }^{*} 3$ genotypes were detected in $5(20 \%), 5(20 \%)$ and $15(60 \%)$ of the 25 graft recipients, respectively. CYP3A5*3 genotypes were found to be a good predictor of tacrolimus Level/Dose (L/D) ratio in kidney transplant recipients. Significantly higher L/D ratios were observed among non-expressors 9.483 $\mathrm{ng} / \mathrm{mL}$ (range $4.5-14.1 \mathrm{ng} / \mathrm{mL}$ ) as compared with the expressors $5.154 \mathrm{ng} / \mathrm{mL}$ (range $4.42-6.5 \mathrm{ng} / \mathrm{mL}$ ) of CYP3A5. Biopsy Proven Acute Rejection (BPAR) episodes were significantly higher in CYP3A5*1 homozygotes compared to patients with CYP3A5*1/*3 and CYP3A5*3/*3 genotypes $(40 \%$ vs. $20 \%$ and $13 \%$, respectively). The dosenormalized tacrolimus concentration $(\mathrm{ng} / \mathrm{mL} / \mathrm{mg} / \mathrm{Kg})$ was significantly lower in patients having CYP $3 A 5^{\star} 1{ }^{*} 3$ polymorphism. This is the first study to extensively determine the effect of CYP $3 A 5^{\star} 3$ genetic polymorphism on tacrolimus pharmacokinetics in South Indian renal transplant recipients, and this study also showed that the majority of our patients carry mutant allele A6986G in the CYP3A5*3 gene. Identification of CYP3A5 polymorphism prior to transplantation is important for selecting the appropriate initial dosage of tacrolimus for each patient.
\end{abstract}

Keywords: Kidney transplant patients; CYP3A5 genotype; Tacrolimus; BPAR

\section{Background}

Tacrolimus is the first line immunosuppressant recommended for renal transplant patients as it provides better allograft survival and lower incidence of nephrotoxicity. It acts through the inhibition of calcineurin via complex formation with immunophilin FK-506 binding protein 12 [1]. However, tacrolimus has a narrow therapeutic index and highly variable pharmacokinetics, and despite tailoring the initial dosage to body weight and monitoring blood concentrations of the drug [2], reaching target blood concentrations is a slow process with a high degree of inter-and intra-individual variability. Therefore, tacrolimus users could be at risk of suffering graft rejection and toxicity. The elimination half-life of tacrolimus has been reported as $19 \mathrm{~h}$ in renal transplant recipients. Less than $1 \%$ of the intravenous dose of tacrolimus is eliminated unchanged in the urine [3]. The whole blood concentration of tacrolimus should be monitored and blood levels should be maintained in the range of 5-15 ug/L for optimal efficacy and minimal toxicity, such as nephrotoxicity and neurotoxicity in renal transplant recipients $[4,5]$.

Genetic factors are believed to contribute to the inter-individual variations in drug administration; in fact, studies have reported that genetics can account for $20-95 \%$ of the variability in drug disposition and effects [6,7]. At the time of administration to the target site, the drugs can interact with hundreds of proteins, including receptors, transporters and metabolizing enzymes. The low tacrolimus bioavailability has been attributed to inter-individual differences in the expression of the metabolizing enzyme CYP3A5 polymorphic genes affecting the quantity or activity of these products [8]. CYP3A is the primary subfamily in humans, responsible for CYP-mediated phase I metabolism of more than $50 \%$ of current administered drugs. The CYP3A5 protein is encoded by CYP3A5 gene [9]. This gene is a part of cluster of CYP genes on chromosome 7q21.1 [10]. Among the CYP3A subfamily, CYP3A5 is the most frequent and functionally significant single nucleotide polymorphism (SNP) in human drug metabolism. This SNP carries a substitution of A to G at position 6986 within intron 3 , which leads to an alternative splicing and truncated protein [11]. 
Citation: Sarasamma S, Gracious N, Nair SS, Radhakrishnan R (2016) Pharmacogenomics of CYP3A5 Polymorphism: Predicting Doseadjusted Trough Levels of Tacrolimus in South Indian Renal Transplant Patients. J Pharmacogenomics Pharmacoproteomics 7: 161. doi:10.417212153-0645.1000161

Page 2 of 5

Based on previous studies done in the West, patients were classified into two categories: (i) Expressors $\left(\mathrm{CYP} 3 \mathrm{~A} 5^{\star} 1 / \mathrm{CYP} 3 \mathrm{~A} 5^{\star} 1\right.$ or CYP3A $5^{\star} 1 /$ CYP3A $5 * 3$ ); and (ii) CYP3A5 non-expressors (CYP3A $5^{\star} 3 /$ CYP3A $5^{\star} 3$ ) [12]. CYP3A5 expressors need higher doses of tacrolimus to reach the same therapeutic levels. As high tacrolimus levels are associated with acute renal failure, genetic variation in CYP3A5 also impacts the risk of developing chronic renal failure.

However, immunosuppression is still unsuccessful in some patients, while causing toxicity in others. Monitoring tacrolimus whole blood concentrations is therefore essential to optimize the therapeutic dose in patients receiving this drug for the prophylaxis of acute rejection after organ transplantation. Several studies have reported that the CYP3A5 polymorphism is associated with tacrolimus dose-adjusted concentrations in many ethnic groups $[13,14]$. However, limited data is available about South Indian renal transplant recipients and the effects of cytochrome P450 3A5 polymorphism on clinical outcomes in the post-transplantation period. Therefore, the objectives of this study included investigation of the implication of CYP3A5 genetic polymorphism correlated with increased tacrolimus exposure on standard dosages and thus might predict lower dose requirements.

\section{Materials and Methods}

\section{Subjects}

Renal transplant (RT) recipients included in this study were on routine follow-up at the Out Patient clinic of the Department of Nephrology, Govt. Medical College Hospital, and Trivandrum, India. Twenty-five unrelated patients who had a single renal allograft and were on immunosuppressive drug tacrolimus for more than three months were sampled for genotype analysis and for estimating blood tacrolimus levels. Clinical data, personal and family history were recorded in a well-designed Performa. Patients taking other medications that may interact with tacrolimus, and patients who were unavailable for follow up were excluded. The study was approved by the Institute Ethics Committee at Rajiv Gandhi Centre for Biotechnology and all patients gave written informed consent to participate in the study.

\section{Immunosuppressive regimen}

The treatment scheme consisted of a combination of a calcineurin inhibitor (tacrolimus) with a purine inhibitor (mycophenolate mofetil) and a steroid (prednisolone). The starting dose of tacrolimus was 0.1 $\mathrm{mg} / \mathrm{kg} / \mathrm{day}$, given twice daily. Then, the dose was adapted to obtain a trough blood concentrations target between 5 and $10 \mathrm{ng} / \mathrm{mL}$. The dose was subsequently adjusted according to the whole blood trough (C0) levels. Body weight, laboratory data (albumin, serum creatinine), tacrolimus dosage $(\mathrm{mg} / \mathrm{kg})$ and $\mathrm{C} 0$ levels were recorded for the first month after transplantation.

\section{Sample collection and DNA extraction}

$4 \mathrm{~mL}$ blood samples were drawn from each patient in a vacutainer tube containing ethylenediaminetetraacetic acid (EDTA). Genomic DNA was extracted from $500 \mu \mathrm{L}$ of whole blood using phenolchloroform method and estimated yields of DNA were determined by measuring the absorbance at $260 \mathrm{~nm}$ (A260). DNA extracts were kept at $-80^{\circ} \mathrm{C}$ for long-term storage and at $4^{\circ} \mathrm{C}$ for short-term storage.

\section{PCR amplification of CYP3A5 genotype}

The molecular genotyping of CYP3A5 A6986G allele was performed by the polymerase chain reaction-restriction fragment length polymorphism approach as described by van Schaik et al. [10] and Macphee et al. [6], respectively, with some modifications.

PCR primers for CYP3A5 were designed to amplify a 293 bp fragment (forward primer: 5'-CATCAGTTAGTAGACAGATGA-3', reverse primer: 5'-GGTCCAAACAGGGAAGAAATA-3'). In each reaction, approximately $100 \mathrm{ng}$ genomic DNA, $1 \mathrm{uM}$ of each forward and reverse primer, and green PCR master mix (Emerald) were used in a final reaction volume of $25 \mu \mathrm{L}$. PCR was performed in a Applied Biosystems thermal cycle (Cycling parameters were initial denaturation at $94^{\circ} \mathrm{C}$ for $30 \mathrm{~s}$, annealing at $56^{\circ} \mathrm{C}$ for $30 \mathrm{~s}$, extension at $72^{\circ} \mathrm{C}$ for $1 \mathrm{~min}$, and a final extension step at $72^{\circ} \mathrm{C}$ for $5 \mathrm{~min}$; the number of cycles was 35 . The PCR product was analyzed on a $2 \%$ agarose EDTA gel with ethidium bromide staining and bands were analyzed by a gel documentation system (Figure 1A).

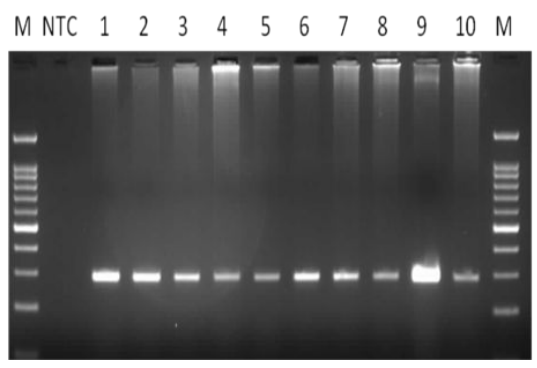

Figure 1A: PCR for CYP3A5. Lane M, base pair marker (100 bp DNA ladder); NTC Negative control; Lanes 1-10 pcr product for CYP3A5 from 10 DNA samples. Analysis on a 2\% agarose EDTA gel.

Enzymatic digestion of amplified PCR products of CYP3A5 was performed using SSPI endonuclease (New England Biolabs). A $20 \mu \mathrm{L}$ aliquot of PCR product was incubated for $2 \mathrm{~h}$ at $37^{\circ} \mathrm{C}$ and subsequently analysed on $4 \%$ agarose/TBE gel with ethidium bromide staining and bands were detected by a short wavelength UV trans-illuminator (Figure 1B).

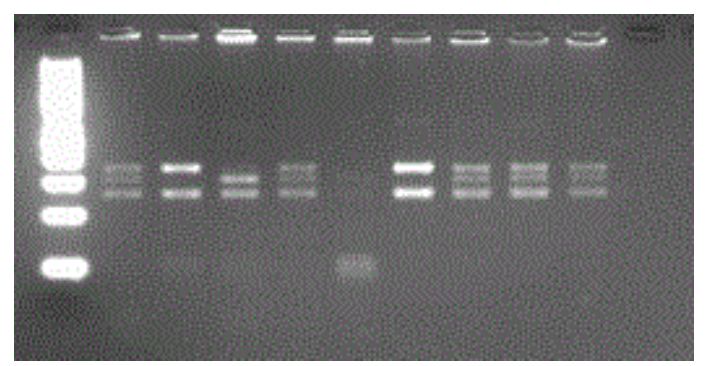

Figure 1B: RFLP for CYP3A5. Lane M, base pair marker (100 bp DNA ladder); Lanes 1-9 SSPI-digested PCR products from 9 PCR products. CYP 3 A $5{ }^{*} 1{ }^{*} 1$ genotype yields 148 -, 125 -, and $20 \mathrm{bp}$ bands. 
Citation: Sarasamma S, Gracious N, Nair SS, Radhakrishnan R (2016) Pharmacogenomics of CYP3A5 Polymorphism: Predicting Doseadjusted Trough Levels of Tacrolimus in South Indian Renal Transplant Patients. J Pharmacogenomics Pharmacoproteomics 7: 161. doi:10.417212153-0645.1000161

Page 3 of 5

SSPI digestion of the 293 bp CYP3A5 fragment yielded fragments of A6986 allele $\left(\mathrm{CYP}^{2} 5^{\star} 1\right.$ ) are $148-, 125-$ and 20 bp bands whereas (CYP3A5*3) genotype gave 168 - and 125 bp bands.

\section{Sequencing of CYP3A5 allelic variants}

We confirmed the presence of variant alleles of CYP3A5 by direct sequencing of the PCR product on an automated ABI 3100 capillary sequencer (Applied Biosystems) using the Big Dye Terminator Cycle Sequencing kit (Applied Biosystems). DNA sequences were compared to the reference sequence (NG_007938.1) stored in the NCBI genome browser (http://www.ncbi.nlm.nih.gov). Sequencing results were analyzed using Mega version 6 software.

\section{Statistical analysis}

Patients were divided into two groups based on their allelic status for CYP3A5-CYP3A $5 * 3 /{ }^{*} 3$ (homozygotes, non-expressors) and CYP $3 \mathrm{~A}^{\star} 1{ }^{\star} 3$ (heterozygotes, expressors). Tacrolimus trough blood levels were determined for all patients on the sixth post-operative day. The statistical significance of differences in the two groups were examined using the independent' $t$ ' test. Statistical analyses were performed using SPSS software version 16; $\mathrm{p}$-values less than 0.05 were considered statistically significant (Figure 2).

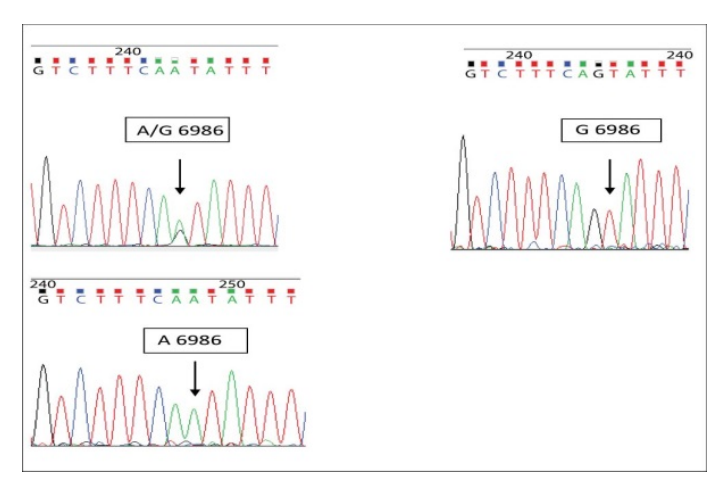

Figure 2: Sequencing results of CYP3A5 A6986G polymorphism.

\section{Results}

\section{Characteristics of study population}

The characteristics of the study population are shown in Table 1. Twenty-five kidney transplant recipients with tacrolimus-based immunosuppressive treatment were included in the study. There were 22 males, and 3 females with a mean $( \pm$ SD) age of $32 \pm 20$ years and mean $( \pm$ SD) body weight of $58 \pm 11 \mathrm{~kg}$. All patients were on tacrolimus-based immunosuppression therapy in combination with mycophenolate mofetil. Three patients received induction therapy; two of them received anti-thymocyte globulin (ATG) and one received basiliximab. Mean $( \pm \mathrm{SD}$ ) donor age was $44 \pm 12$ years. At one month post-transplantation, the incidence of Biopsy Proven Acute Rejection (BPAR) was $20 \%$, all of which were T-cell mediated rejections. Eight biopsies were obtained from the study population in the first month and the incidence of tacrolimus nephrotoxicity in this subgroup was $8 \%$. Gender wise distribution of patients showed that the prevalence of kidney failure was found more often in males (88\%) than in females (12\%).

\begin{tabular}{|l|l|}
\hline Demographic Characteristics & Number \\
\hline Renal transplant recipients & 25 \\
\hline Age at transplantation (years) & $32 \pm 20$ \\
\hline Gender (male/female) & $22 / 3$ \\
\hline Body weight (kg) & $58 \pm 11$ \\
\hline Primary kidney disease & 14 \\
\hline Unknown & 9 \\
\hline Diabetic nephropathy & 2 \\
\hline All values are expressed as mean \pm SD & \\
\hline
\end{tabular}

\section{Frequencies of CYP3A5 genotype}

The CYP $3 \mathrm{~A} 5{ }^{*} 1 /{ }^{*} 1,{ }^{*} 1 /{ }^{*} 3$ and ${ }^{*} 3 /{ }^{*} 3$ genotypes were detected in 5 (20\%), $5(20 \%)$ and $15(60 \%)$ of the 25 graft recipients, respectively. Mean tacrolimus level in the CYP3A $5{ }^{*} 1 /{ }^{\star} 1$ group was $5.154 \mathrm{ng} / \mathrm{mL}$ (range $4.42-6.5 \mathrm{ng} / \mathrm{mL}$ ), the mean for the CYP3A $5^{\star} 1{ }^{\star} 3$ group was $5.348 \mathrm{ng} / \mathrm{mL}$ (range $3.1-9.87 \mathrm{ng} / \mathrm{mL}$ ), and the mean for the CYP $3 A 5^{\star} 3 /{ }^{*}$ group was $9.483 \mathrm{ng} / \mathrm{mL}$ (range $4.5-14.1 \mathrm{ng} / \mathrm{mL}$ ). Therapeutic drug monitoring showed that the mean tacrolimus blood concentration was significantly lower up to the first posttransplantation month for individuals possessing CYP $3 \mathrm{~A}^{\star}{ }^{\star}$.

\section{Effect of CYP3A5 genetic polymorphisms on acute rejection episodes and tacrolimus nephrotoxicity}

BPAR obtained at one month post-transplantation was compared among the three CYP3A5 genotype groups. Acute rejection episodes were significantly higher in CYP $3 \mathrm{~A} 5^{\star} 1$ homozygotes compared to patients with CYP 3 A $5{ }^{\star} 1 /{ }^{\star} 3$ and CYP 3 A $5{ }^{\star} 3 /{ }^{\star} 3$ genotypes ( $40 \%$ vs. $20 \%$ and $13 \%$, respectively). The relationship between CYP3A5 genetic polymorphism and biopsy proven nephrotoxicity due to tacrolimus use was also examined. Eight renal biopsies were obtained during the first month of therapy, of which 2 had evidence of calcineurin inhibitor (CNI) toxicity, both were non-expressors.

\section{Discussion}

To the best of our knowledge, this is the first pharmacogenomic study to explore the relationship between CYP3A5 genotype $(n=25)$ in South Indian renal transplant patients and tacrolimus trough levels. A significant association was found between tacrolimus trough level and CYP3A5 genotype. This finding is in accordance with previous studies $[6,10]$ in other patient populations that showed higher tacrolimus trough levels among kidney transplant patients who were nonexpressors of CYP3A5 (CYP3A5*3/*3).

The ability to predict a patient's drug response based on their genetic information is emerging as a method to reduce adverse events and improve therapeutic efficacy. Targeting therapies to patients who are most likely to benefit while minimizing adverse effects will improve patient care and facilitate the approval of new, innovative drugs. Renal transplantation has largely benefited from tacrolimus therapy, but despite the good clinical results, its management still requires adoption of methods to discriminate therapeutic from toxic effects [12]. The CYP3A5 gene has 11 different polymorphisms, which have been identified to date. CYP3A5 (A6986G) polymorphism (rs 776746) is 
Citation: Sarasamma S, Gracious N, Nair SS, Radhakrishnan R (2016) Pharmacogenomics of CYP3A5 Polymorphism: Predicting Doseadjusted Trough Levels of Tacrolimus in South Indian Renal Transplant Patients. J Pharmacogenomics Pharmacoproteomics 7: 161. doi:10.417212153-0645.1000161

Page 4 of 5

most extensively studied and it is characterized by adenine (A) to guanine $(\mathrm{G})$ substitution at position 6986 within intron 3 of the CYP3A5 gene.

We chose to study this specific genetic polymorphism of CYP3A5 metabolizing enzyme as this critically affects the metabolism of tacrolimus, and this polymorphism is frequently observed in the South Indian population. In our study, we evaluated the effect of CYP3A5 genetic polymorphism on tacrolimus daily dose requirements, in a cohort of kidney transplant recipients. Our results also showed that carriers of at least one active allele $\left(\mathrm{CYP} 3 \mathrm{~A} 5^{\star} 1\right)$ needed significantly higher doses of tacrolimus compared to patients homozygous for $\mathrm{CYP} \mathrm{A}^{*} 3$ (CYP3A5 non-expressors). These results led us to hypothesize those carriers of CYP $3 \mathrm{~A} 5^{\star} 1$ allele has high levels of CYP3A5 expression and enzymatic activity, necessitating higher daily doses in these patients to achieve therapeutic trough levels of tacrolimus. These results have been previously been replicated in other patient populations in the literature for this polymorphism [15].

A previous study by Mohan et al. demonstrated the influence of CYP3A5 polymorphism on tacrolimus drug dosing in North Indian renal allograft recipients. To our knowledge, this is the first investigation to show the association between CYP3A5 genetic polymorphism and tacrolimus drug level in the South Indian population [16].

This study revealed the effect of genetic polymorphisms of CYP3A5 on tacrolimus daily dose requirements and $\mathrm{L} / \mathrm{D}$ ratio in a cohort of South Indian renal transplant patients. The patients with CYP $3 \mathrm{~A}^{\star} 1$ allele required a higher dose of tacrolimus compared to patients homozygous for CYP3A $5{ }^{\star} 3$ (CYP3A 5 non-expressors).

Furthermore, it is hypothesized that the increased activity of CYP3A5 enzyme and the higher tacrolimus dose might induce nephrotoxicity by increasing the level of nephrotoxic tacrolimus metabolites. This hypothesis supports the result that there is increased risk of biopsy proven acute rejection during the first month posttransplantation as patients with $\mathrm{CYP} 3 \mathrm{~A} 5^{*} 1{ }^{*} 1$ genotype had higher risk of developing acute graft rejection episodes compared to CYP $3 A 5^{\star} 3$ homozygote's. This observation is in agreement with the fact that carriers of the wild-type allele $\left(\mathrm{CYP} 3 \mathrm{~A} 5^{\star} 1\right)$ have higher levels of CYP3A5 expression, higher metabolic clearance of tacrolimus, low trough concentrations of tacrolimus, and a higher incidence of acute rejection.

A previous study by Lina et al. [15] demonstrated that CYP3A $5{ }^{\star} 1$ homozygotes were associated with increased risk of acute rejection episodes compared to patients with CYP $3 \mathrm{~A} 55^{\star} 1 / * 3$ and CYP $3 \mathrm{~A} 5 * 3 / * 3$ genotypes ( $38 \%$ vs. $10 \%$ and $9 \%$, respectively, $\mathrm{P}=0.01$ ). These authors had also pointed out that few rejection episodes occurred after the first month of transplantation, so the overall rejection episodes were more crucial during the first month after transplantation [15]. Interestingly, these observations show the importance of adapting tacrolimus daily doses in the early period post-transplantation, where there is a greater risk of developing acute rejection episodes [17-24].

The findings of this study also suggest an association between CYP3A5 genotype and biopsy proven tacrolimus nephrotoxicity. We found increased occurrence of nephrotoxicity in CYP3A5 nonexpressors. This was expected in view of the high trough blood levels in these patients. The present study was limited because of the small number of biopsies taken to substantiate this observation. However, a previous study by Lina Quteineh et al. found no correlation regarding the development of tacrolimus-related nephrotoxicity and CYP3A5 genetic polymorphism [12].

\section{Conclusion}

In conclusion, our study confirms that $\mathrm{CYP} 3 \mathrm{~A} 55^{\star} 3$ genetic polymorphism is significantly associated with tacrolimus concentration in South Indian renal transplantation recipients. Additionally, the study highlights the need to determine each patient's CYP3A5 genotype as a needed part of clinical optimization of tacrolimus dose and to avoid possible incidence of toxicity, since it has been clearly shown that $\mathrm{CYP} 3 \mathrm{~A} 5^{\star} 3 /{ }^{*} 3$ individuals have higher tacrolimus concentrations. We also recommend that a comprehensive study with a larger sample size will strengthen this association. In line with this contention, individualization of medical treatment could result in a great improvement in therapeutic efficacy and reduction of side effects, as well as the reduction of the cost of medical treatment. The tacrolimus concentration could be maintained at different levels according to CYP3A5 genotype condition without frequent therapeutic drug monitoring. Therefore, this data would be helpful to physicians so that by knowing the genotype of the patient before undergoing transplantation they would be able to decide upon the starting dose of tacrolimus so as to avoid high trough levels and consequent nephrotoxicity.

\section{Authors' Contributions}

Sarasamma S: Participation in study design, sample collection, genotyping, manuscript drafting, critical decision and manuscript corrections.

Gracious N: Supervision study design and critical decision.

Nair SS: Statistical analysis.

\section{Acknowledgements}

We acknowledge Dr. Jisa for her assistance with statistical analysis and Dr. Sara Jones and Dr. Iype Joseph for thoughtful suggestions. The authors gratefully acknowledge the participation of the renal transplant recipients of the Department of Nephrology, Govt. Medical College Hospital, Thiruvananthapuram, and Kerala, India.

\section{Funding}

This work has been supported by the Department of Biotechnology, New Delhi, India.

\section{References}

1. Fruman DA, Bierer BE, Benes JE, Burakoff SJ, Austen KF, et al. (1995) The complex of FK506-binding protein 12 and FK506 inhibits calcineurin phosphatase activity and IgE activation - induced cytokine transcripts, but not exocytosis, in mouse mat cells. J Immunol 154: 1846-1851.

2. Bowman LI, Brennan DC (2008) The role of tacrolimus in renal transplantation. Expert Opin Pharmacother 9: 635-643.

3. Peters DH, Fitton A, Plosker GL, Faulds D (1993) Tacrolimus. A review of its pharmacology, and therapeutic potential in hepatic and renal transplantation. Drugs 46: 746-794.

4. Meier-Kriesche HU, Li S, Gruessner RW, Fung JJ, Bastami RT, et al. (2006) Immunosuppression: evolution in practice and trends, 1994-2004. Am J Transplant 6: 1111-1131. 
Citation: Sarasamma S, Gracious N, Nair SS, Radhakrishnan R (2016) Pharmacogenomics of CYP3A5 Polymorphism: Predicting Doseadjusted Trough Levels of Tacrolimus in South Indian Renal Transplant Patients. J Pharmacogenomics Pharmacoproteomics 7: 161. doi:10.417212153-0645.1000161

Page 5 of 5

5. Jusko WJ, Thomson AW, Fung J, McMaster P, Wong SH, et al. (1995). Consensus document: therapeutic monitoring of tacrolimus (FK 506). Ther Drug Monit 17: 606-614.

6. Macphee IA, Fredericks S, Tai T, Syrris P, Carter ND, et al. (2002) Tacrolimus Pharmacogenetics: polymorphisms associated with expression of cytochrome P4503A5 and P-glycoprotein correlate with dose requirement. Transplantation 74: 1486-1489.

7. Anglicheau D, Flamant M, Schlageter MH, Martinez F, Cassinat B, et al. (2003) Pharmacokinetic interaction between corticosteroids and tacrolimus after renal transplantation. Nephrol Dial Transplant 118: 2409-2414.

8. de Jonge $\mathrm{H}$, Kuypers DR (2008) Pharmacogenetics in solid organ transplantation: current status and future directions. Transplant Rev(Orland) 22: 6-20.

9. Shiraga T, Matsuda H, Nagase K, Iwasaki K, Noda K, et al. (1994) Metabolism of FK 506 a potent immunosuppressive agent, by Cytochrome p450 enzymes in rat, dog an human liver microsomes. Biochem Pharmacol 47: 727-725.

10. van Schaik RH, van der Heiden IP, van den Anker JN (2002) CYP3A5 variant allele frequencies in Dutch Caucasians. Clin Chem 48: 1668-1671.

11. Hebert MF (1997) Contributions of hepatic and intestinal metabolism and P-glycoprotein to cyclosporine and tacrolimus oral drug delivery. Adv Drug Deliv Rev 27: 201-214.

12. Halloran PF (2004) Immunosuppressive drugs for kidney transplantation. N Engl J Med 351: 2715-2729.

13. Staaz CE, Tett SE (2005) Pharmacokinetic considerations relating to tacrolimus dosing in the elderly. Drugs aging 22: 541-557.

14. Provenzani A, Notarbartolo M, Labbozzetta M, Poma P, Vizzini G, et al. (2011) Influence of CYP3A5 and ABCB1 polymorphism and other factors on tacrolimus dosing in Caucasian liver and Kidney transplant patients. Int J Mol Med 28: 1093-1102.
15. Kuehl P, Zhang J, Lin Y, Lamba J, Assem M, et al. (2001) Sequence diversity in CYP3A promoters and characterization of the genetic basis of polymorphic CYP3A5 expression. Nat Genet 27: 383-391.

16. Hustert E, Haberl M, Burk O, Wolbold R, He YQ, et al. (2001) The genetic determinants of the CYP3A5 polymorphism. Pharmacogenetics 11: 773-779.

17. MacPhee IA, Fredericks S, Tai T, Syrris P, Carter ND, et al. (2004) The influence of pharmacogenetics on the time to achieve target tacrolimus concentrations after kidney transplantation. Am J Transplant 4: 914-919.

18. Barry A, Levine M (2010) A systematic review of the effect of CYP3A5 genotype on the apparent oral clearance of tacrolimus in renal transplant recipients. Ther Drug Monit 6: 708-714.

19. Dai Y, Hebert MF, Isoherranen N, Davis CL, Marsh C, et al. (2006) Effect of CYP3A5 polymorphism on tacrolimus metabolic clearance in vitro. Drug Metab Dispos 34: 836-847.

20. Patel M, Gumber M, Kute V, Shah P, Patel H, et al. (2014) Influence of CYP3A5 polymorphism on tacrolimus drug dosing in Indian renal allograft recipients: initial experience. Molecular Cytogenetics 7: 98.

21. Kamdem LK, Streit F, Zanger UM, Brockmoller J, Oellerich M, et al. (2005) Contribution of CYP3A5 to the in vitro hepatic clearance of tacrolimus. Clin Chem 8: 1374-1381.

22. Benet LZ (1998) Impact of intraindividual variability of drugs on therapeutics outcomes: lessons from outside transplantation. Transplant Proc 30: 1650-1651.

23. Thervet E, Anglicheau D, King B, Schlageter MH, Cassinat B, et al. (2003) Impact of cytochrome P450 3A5 genetic polymorphism on tacrolimus doses and concentration-to-dose ratio in renal transplant recipients. Transplantation 76: 1233-1235.

24. Thummel KE (2004) A genetic test for immunosuppressant dose selection. Pharmacogenetics 14: 145-146. 